\title{
3-D Visualization on Workspace of Parallel Manipulators
}

\author{
Yoshito TANAKA ${ }^{* *}$, Isao YOKOMICHI** Junko ISHII $^{* *}$ \\ and Toshiaki MAKINO ${ }^{* * *}$ \\ **Department of Control and Information Systems Engineering, \\ Kitakyusyu National College of Technology, \\ 5-20-1 Shii, Kokuraminami-ku, Kitakyusyu-shi, Fukuoka 802-0985, Japan \\ E-mail:stanaka@kct.ac.jp

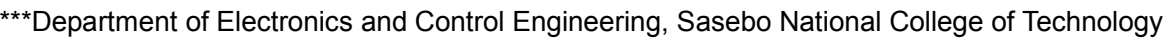 \\ 1-1 Okishinmachi, Sashebo, Japan
}

\begin{abstract}
In parallel mechanisms, the form and volume of workspace also change variously with the attitude of a platform. This paper presents a method to search for the workspace of parallel mechanisms with 6-DOF and 3D visualization of the workspace. Workspace is a search for the movable range of the central point of a platform when it moves with a given orientation. In order to search workspace, geometric analysis based on inverse kinematics is considered. Plots of 2D of calculations are compared with those measured by position sensors. The test results are shown to have good agreement with simulation results. The workspace variations are demonstrated in terms of $3 \mathrm{D}$ and $2 \mathrm{D}$ plots for prototype mechanisms. The workspace plots are created with OpenGL and Visual C++ by implementation of the algorithm. An application module is developed, which displays workspace of the mechanism in 3D images. The effectiveness and practicability of 3D visualization on workspace are successfully demonstrated by 6-DOF parallel mechanisms.
\end{abstract}

Key words: Workspace, Parallel Mechanisms, 3D-Visualization, OpenGL, Inverse-Kinematics, Application Module

\section{Introduction}

Parallel kinematic manipulators offer several advantages over their counterparts for certain applications. Among the advantages are greater load carrying capacities, higher stiffness, and reduced sensitivity to certain errors. Stewart type of parallel mechanisms is one of parallel manipulators that are used increasingly in manufacturing, inspection and research ${ }^{(1),(2)}$. However, these machine's non-intuitive kinematics, workspace, and error characteristics create obstacles to industry's acceptance ${ }^{(3)}$. As to the workspace restrictions due to limited strut lengths have been considered based on geometrical properties of the workspace ${ }^{(4)}$. Other important constraints, such as joint angles and avoiding strut collisions were integrated into workspace modeling to verify the trajectory within the workspace.

In this paper, geometrical analysis for computing the workspace of 6-DOF Stewart manipulators is presented using inverse kinematics when only the constraints on the strut limits are considered. In practice, the workspace producing large range of orientation can be regarded as being useful; hence the workspace has been examined for desired orientations with large rotation angles of the platform. In the workspace analysis $2 \mathrm{D}$ plots of boundary curves in XY plane are used to construct the whole workspace. To confirm the real-time visualization of the workspace and to understand the motion capabilities of a prototype machine, an application module is developed which simultaneously displays motions and 
workspace of the manipulator in $3 \mathrm{D}$ images. Since the motion and the workspace are too complex to gain an immediate understanding of their correlation, the module may be useful to create the spatial motion of the virtual prototype machine and estimate a real-time workspace boundary.

\section{Workspace Analysis}

\subsection{Kinematics of the Parallel Manipulator}

The workspace of the parallel manipulator can be described as the set of all spatial coordinate points of the center of the platform with a given orientation of the platform. The workspace of parallel manipulators is limited owing to three types of constraints: 1) mechanical limit on the passive joints; 2) interference between links; and 3) limited range in the length of linear actuators (cylinders). The prototype (Fig.1) has no mechanical limits on joints and interference between links within the range in the length of cylinders. Hence, the geometrical analysis for computing the workspace will be described when only the length limits of cylinders are considered.

The parallel manipulator, whose design is based on the Stewart Platform mechanisms, comprises a platform, six cylinders and a base. Six cylinders, each of which is composed of

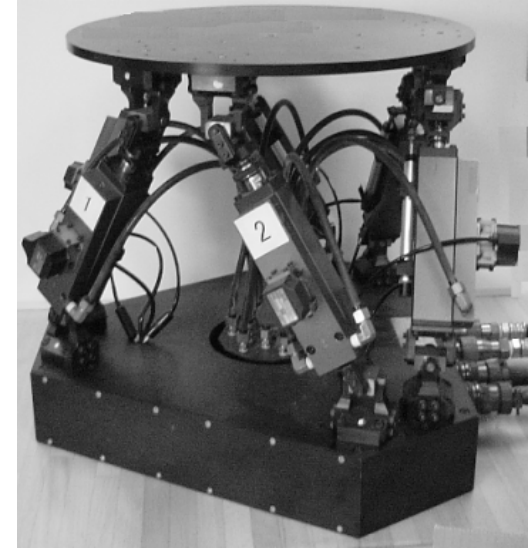

Fig.1 Stewart platform-based manipulator

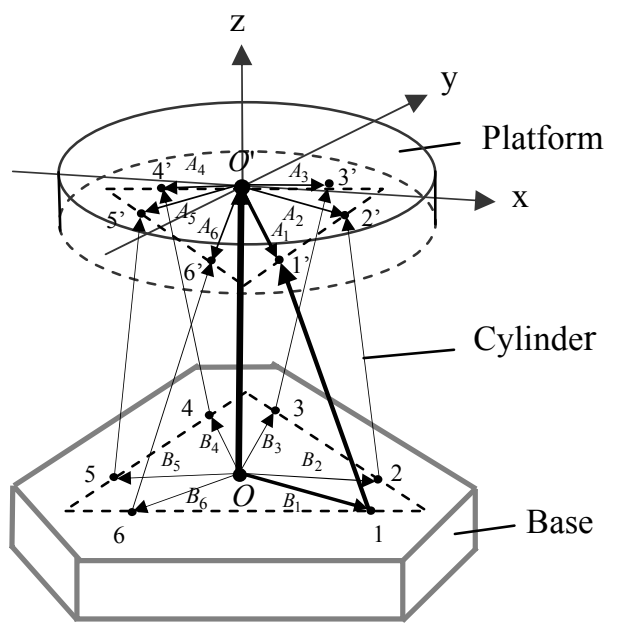

Fig.2 Platform frame assignment

a hydraulic actuator, link the platform and base together. Driving the servo to shorten or extend cylinder lengths produces motion of the platform. The 6-DOF reference is transformed into desired cylinder lengths using inverse kinematics, which is compared with the cylinder lengths measured by LVDT sensors.

This section will use vector algebra to attain a closed-form solution for the inverse kinematics. The inverse kinematics of the parallel manipulator can be formulated so as to determine the required cylinder vector for a given orientation of the platform with respect to the base. Fig. 2 shows that two coordinate frame $\{A\}$ and $\{B\}$ are assigned to the platform

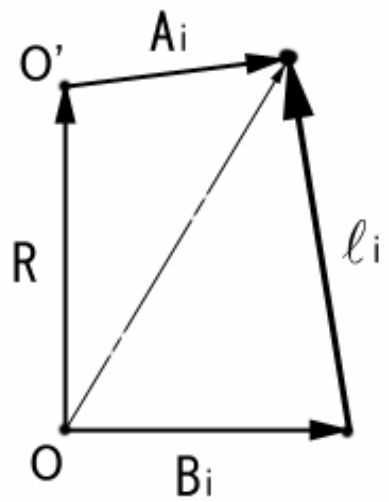

Fig.3 Vector diagram for the i-th cylinder 
and the base of the manipulator, respectively. The origin of frame $\{\mathrm{A}\}$ is at the centroid $\mathrm{O}$ ' of the platform, while the frame $\{\mathrm{B}\}$ has its origin at the centroid $\mathrm{O}$ of the base. The pose of the platform is specified by rotation of frame $\{A\}$ and the position of the centroid $\mathrm{O}^{\prime}$ with respect to the frame $\{\mathrm{B}\}$. We proceed to consider the vector diagram for the $\mathrm{i}$-th cylinder given in Fig.3. The position of the centroid $O^{\prime}$ is represented by vector $R=(x, y, z)^{T}$ with respect to frame $\{\mathrm{B}\}$. Furthermore, if vector $\mathrm{Ai}$ describes the position of the attachment point of the cylinder on the platform with respect to frame $\{B\}$, and vector $\mathbf{B} i$ the position of the attachment point on the base, then the cylinder vector and its Euclidean norm expressed with respect to frame $\{\mathrm{B}\}$ can be computed by

$$
\begin{aligned}
& \ell_{i}=A_{i m} T+R-B_{i} \\
& L_{i}=\left\|\ell_{i}\right\|=\left\|A_{i m} T+R-B_{i}\right\|
\end{aligned}
$$

where $\mathbf{A}_{\text {im }}$ describes the attachment point of the cylinder on the platform relative to frame $\{A\}$ and $T$ represents the orientation matrix of the platform which can be expressed with roll-pitch-yaw angles as $\phi, \theta$, and $\varphi$ by

$$
T=\left(\begin{array}{ccc}
1 & 0 & 0 \\
0 & \cos \phi & \sin \phi \\
0 & -\sin \phi & \cos \phi
\end{array}\right)\left(\begin{array}{ccc}
\cos \theta & 0 & -\sin \theta \\
0 & 1 & 0 \\
\sin \theta & 0 & \cos \theta
\end{array}\right)\left(\begin{array}{ccc}
\cos \varphi & \sin \varphi & 0 \\
-\sin \varphi & \cos \varphi & 0 \\
0 & 0 & 1
\end{array}\right)
$$

Equations (1a) and (1b) represent the closed-form solution to the inverse kinematics problem in the sense that required cylinder length $\mathrm{Li}=\{\mathrm{L}\} \quad(\mathrm{i}=1,2 \ldots 6)$ can be computed to yield a given pose of frame $\{A\}$.

\subsection{Numerical Methods}

The solution of the inverse kinematic problem developed above can be used to describe the workspace of the parallel manipulator. If the mechanical interference is neglected, the boundary of the workspace is attained whenever at least one of the cylinders reaches one of its limits. If the range of motion of the cylinders given by Eq. (1b) satisfies

$$
L_{\min }<L_{i}<L_{\max }, i=1,2, \cdots, 6
$$

then vector $\mathbf{R}$ can describe the workspace for a specified platform orientation. A section of the workspace can be obtained on planes parallel to the xy plane as show in Fig. 4.

For a $x_{j} y_{j}$ plane with $z=z_{j}$, vector $\mathbf{R}(i, j)$ is defined in the form

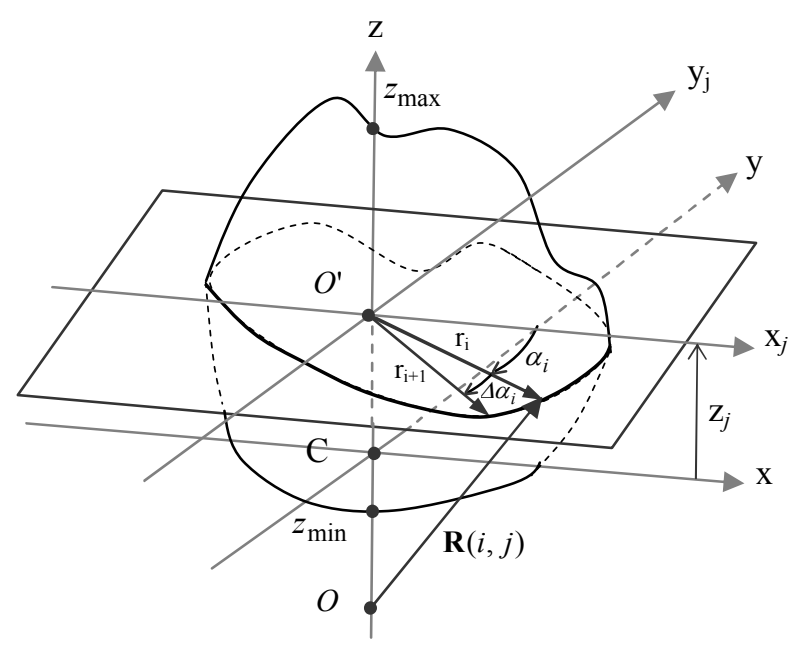

Fig.4 Determination of the boundary in xy plane 


$$
R(i, j)=\left(r_{i} \cos \alpha_{i}, r_{i} \sin \alpha_{i}, z_{j}+R_{0}\right)
$$

where $r_{i}$ and $\alpha_{i}$ are treated as a polar coordinate, and $\mathrm{R}_{0}$ is the distance between O-O' when the pose of the platform is neutral position. The process of searching the boundary of workspace on $\mathrm{x}_{\mathrm{j}} \mathrm{y}_{\mathrm{j}}$ plane is accomplished by rotating the radius $\mathrm{r}_{\mathrm{i}}$ for every finite interval: $0 \leq \alpha_{i} \leq 2 \pi$, and $z_{\min } \leq z_{j} \leq z_{\max }$ using relations (1b), (2), and (4). The algorithm is the following. Taking a polar angle $\alpha_{i}$ in the section and drawing a polar line $r_{i}$, the intersection point between the line $r_{i}$ and the boundary curve of the workspace in this section can be obtained.

\subsection{Geometric Description of the Workspace}

The required cylinder lengths can be directly computed from Eq. (1b) for given values of the position of joints on the base and on the platform, and for prescribed values of a pose of the platform. If the range of motion of the cylinders is given by

$$
L_{\min } \leq L_{i} \leq L_{\max }
$$

then the boundary of the workspace can be obtained geometrically as set of points with $\mathrm{L}_{\mathrm{i}}=\mathrm{L}_{\min }$ or $\mathrm{L}_{\mathrm{i}}=\mathrm{L}_{\max }$ which represent concentric spheres of radii $\mathrm{L}_{\min }$ and $\mathrm{L}_{\max }{ }^{(7)}$. Hence, the workspace of the parallel manipulator can be described as the intersection of 6 regions, so each of these regions is the difference of two concentric spheres. If we desire to find the section of the workspace on a plane parallel to the $x y$ plane defined as $z=z_{j}(z-l e v e l$ cutting plane), then the regions will be 6 pairs of concentric circles. The position of the centers of the circles will depend on the orientation specified for the platform and on the joint positions.

Table 1 Geometric properties of the prototype manipulator

\begin{tabular}{|c|c|c|c|}
\hline $\mathrm{A}_{1}$ & $(119.8127 .5,-60.0)^{\mathrm{T}}$ & $\mathrm{B}_{1}$ & $(221.4, \quad 40.0, \quad 50.0)^{\mathrm{T}}$ \\
\hline $\mathrm{A}_{2}$ & $(50.5,167.5,-60.0)^{\mathrm{T}}$ & $\mathrm{B}_{2}$ & $(-76.0, \quad 211.7, \quad 50.0)^{\mathrm{T}}$ \\
\hline $\mathrm{A}_{3}$ & $(-170.3,40.0,-60.0)^{\mathrm{T}}$ & $\mathrm{B}_{3}$ & $(-145.3, \quad 171.7, \quad 50.0)^{\mathrm{T}}$ \\
\hline $\mathrm{A}_{4}$ & $(-170 .,-40.0,-60.0)^{\mathrm{T}}$ & $\mathrm{B}_{4}$ & $\left(\begin{array}{lll}-145.3, & -171.7, & 50.0\end{array}\right)^{\mathrm{T}}$ \\
\hline $\mathrm{A}_{5}$ & $(50.5,-167.5,-60.0)^{\mathrm{T}}$ & $\mathrm{B}_{5}$ & $\left(\begin{array}{lll}-76.0, & -211.7, & 50.0\end{array}\right)^{\mathrm{T}}$ \\
\hline $\mathrm{A}_{6}$ & $(119 .-127.5,-60.0)^{\mathrm{T}}$ & $\mathrm{B}_{6}$ & $(221.4, \quad-40.0, \quad 50.0)^{\mathrm{T}}$ \\
\hline $\mathrm{L}_{\max }$ & 353.0 & $\mathrm{~L}_{\min }$ & 255.0 \\
\hline
\end{tabular}
(all length are given in $\mathrm{mm}$ )

The workspace of the manipulator shown in Fig.1 will now be studied. Its geometric properties are summarized in Table1. Figures 5, 6, 7 and 8 depict the trajectories of six cylinders in $\mathrm{z}$-level cutting planes ( $\mathrm{z}=0$ ), (Fig. (a)) for reference orientations, as well as the corresponding workspace boundaries for various z-level cutting planes (Fig. (b)). Figures 5-8 (a) indicate how each of the lower and upper limits of the cylinder lengths contributes the workspace boundaries, depending on the orientations of the platform. Since all the inner and outer regions of the circular arcs defined, respectively by $\mathrm{Li}_{\max }$ and $\mathrm{Li}_{\min }$, comprise the reachable space (the enclosed areas), the circular arcs located inside all the internal $\left(\mathrm{Li}_{\max }\right)$ and external circles $\left(\mathrm{L}_{\min }\right)$ constitute the outside boundary of the workspace.

It is noted that the boundaries of the workspace are depend only on circular arcs of $\operatorname{six} \mathrm{L}_{\max }$ 's for the three reference orientations, except one (Fig.6 (a)) where the outer circular arcs of $\mathrm{L} 5_{\min }$ contributes the boundary of the workspace. On the other hand, the level curve of the workspace boundary away from neutrality level $(\mathrm{z}=0)$ varies in shape and area, due to the constraints of lower limits of cylinder lengths Li $_{\min }$ (Figs.5-8 (b)). 


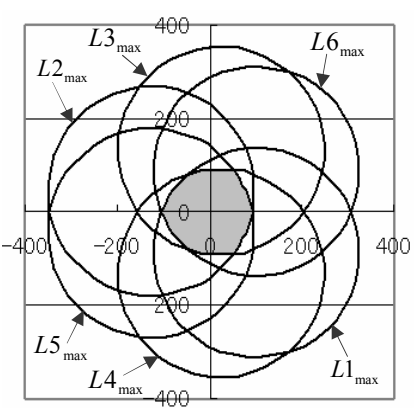

(a) Trajectory of 6 cylinders

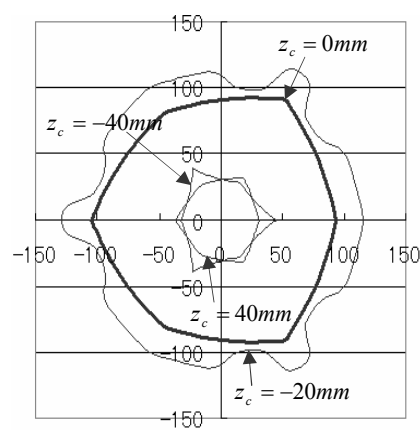

(b) Workspace boundary

Fig.5 2D Plots of workspace (Neutrality)

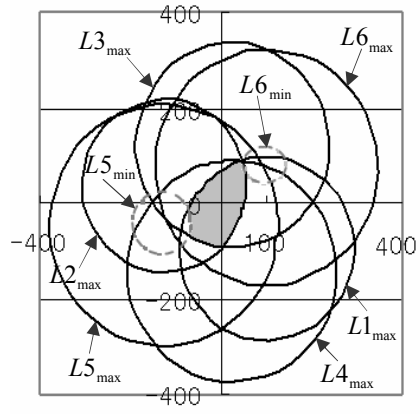

(a) Trajectory of 6 cylinders

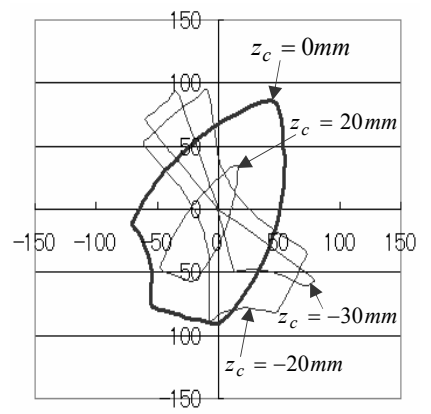

(b) Workspace boundary

Fig.6 2D Plots of workspace (roll: $\phi=10^{\circ}$ )

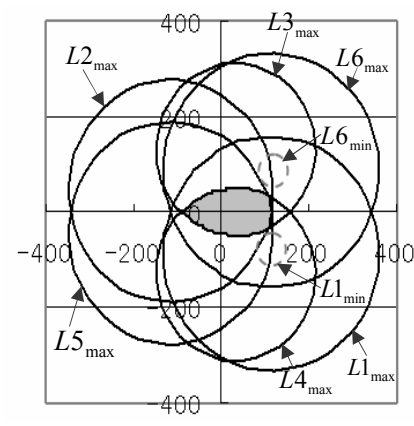

(a) Trajectory of 6 cylinders

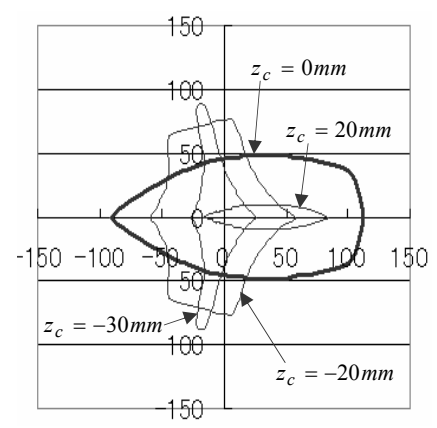

(b) Workspace boundary

Fig.7 2D Plots of workspace (pitch: $\theta=10^{\circ}$ )

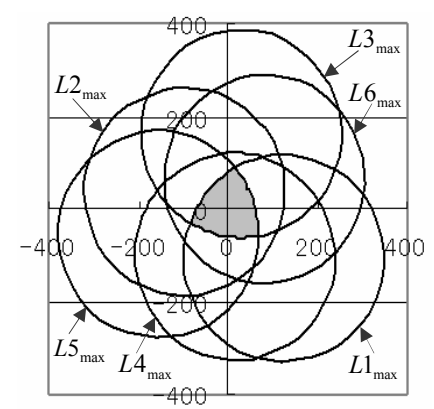

(a) Trajectory of 6 cylinders

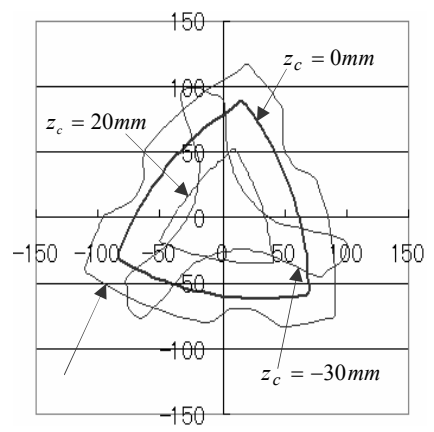

(b) Workspace boundary

Fig.8 2D Plots of workspace (yaw: $\varphi=10^{\circ}$ ) 


\section{Experimental tests of workspace}

\subsection{Method of Measurement}

To examine the validity of the workspace derived via geometrical model, two-dimensional measurements were conducted with the prototype of parallel mechanisms. Figure 9 shows the experimental apparatus and its setup. The LED is placed on the center of platform of which position can be measured with PSD camera via emission signal of the LED. The controller receives the signal of PSD camera to make 2D measurement data, which are transferred to $\mathrm{PC}$ for the calculation of the displacements of the center of platform. The PSD camera looks down the surface of the platform, as shown in Fig.8, which causes the distorted position of the LED. To correct the distortions, one needs to use the factor that can provide the real information of the center position of platform. The voltage signal monitored by PSD camera was transformed into the data corresponding to the real center position of platform using the factor.

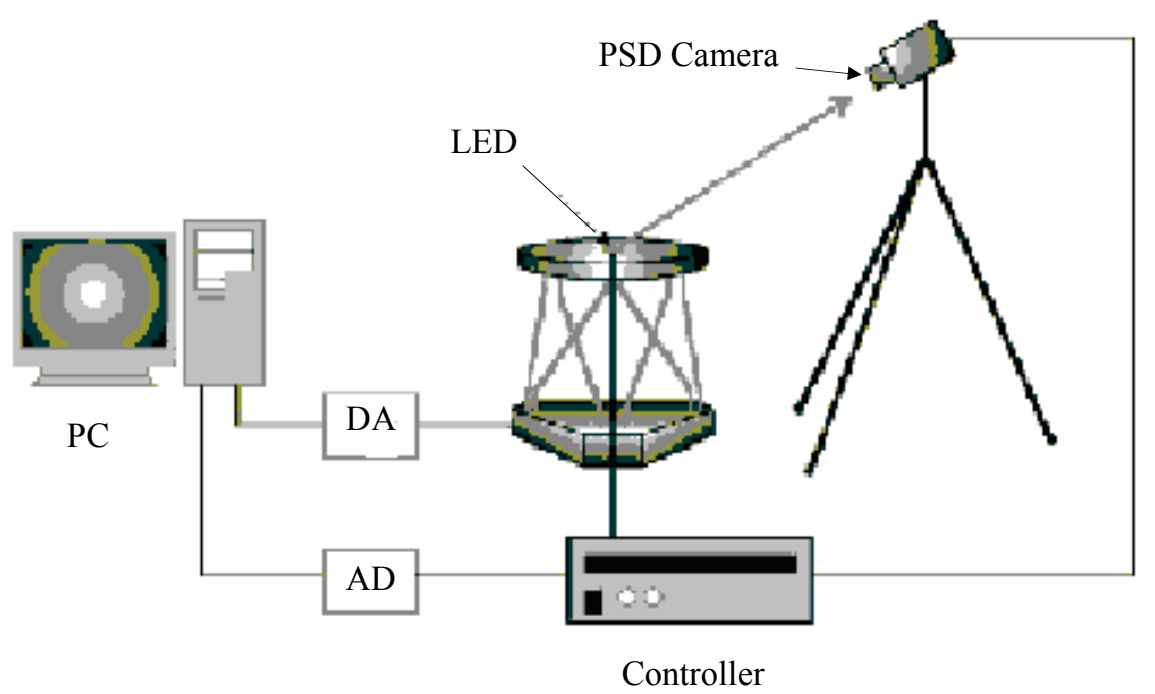

Fig.9 Experimental apparatus

\subsection{D display and measurements}

Figure 10 compares the geometrical workspace and measurements on the $x-y$ plane when the platform rolls at $\varphi=10$ degrees. Figures $10(a)$, (b), and (c) corresponds $z_{i}=10,0$, and $-10 \mathrm{~mm}$, respectively. It is shown that the geometrical results agree very well with the measurements in terms of both the shape and the dimension of the workspace. Also, the difference between measurements and geometrical results is within $5 \%$ for the maximum movements of the platform. It is also noted that the workspace of geometrical method corresponds well with measurements for the case of $y$ and z-axis rotation. In view of the preceding experiments, this geometrical treatment confirmed the validity of the analysis of the workspace when there is a geometrical constraint on the cylinder actuator.

\section{3D display of workspace of manipulator}

\subsection{Construction of 3D display program system}

The above presented algorithms for estimating the workspace of the parallel manipulator were first implemented in the $\mathrm{C}++(\mathrm{VC}++)$ programming language and tested to graphically display the motion and workspace using OpenGL.

The program was then defined as DLL function that executes the operation shown in Fig.11. The DLL was incorporated and declared into the Visual Basic (VB) program as a visualization tool that simulates the motion of the mechanism and displays its workspace simultaneously. 


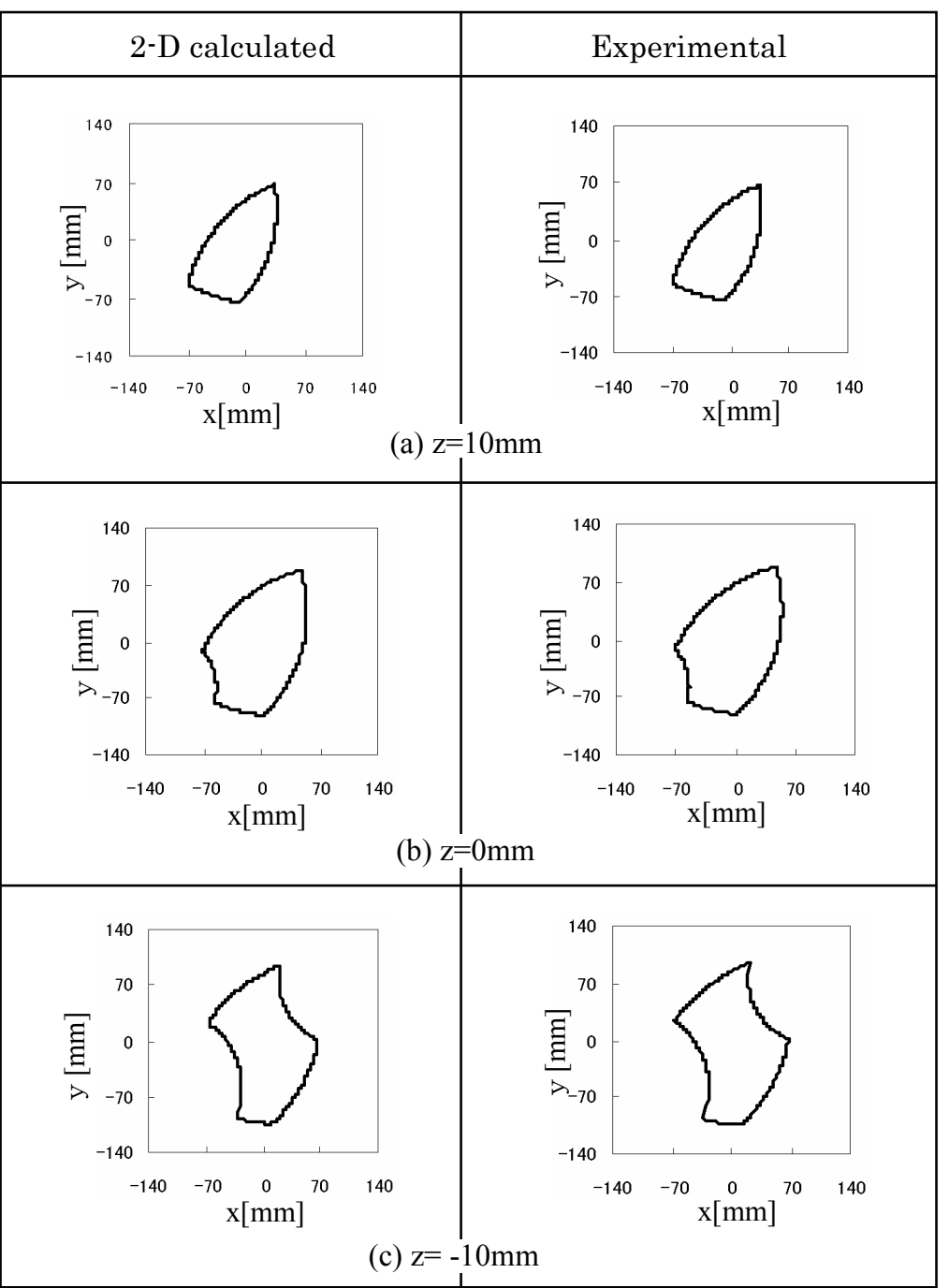

Fig.10 Comparison between calculation and measurements (roll: $\phi=10^{\circ}$ )

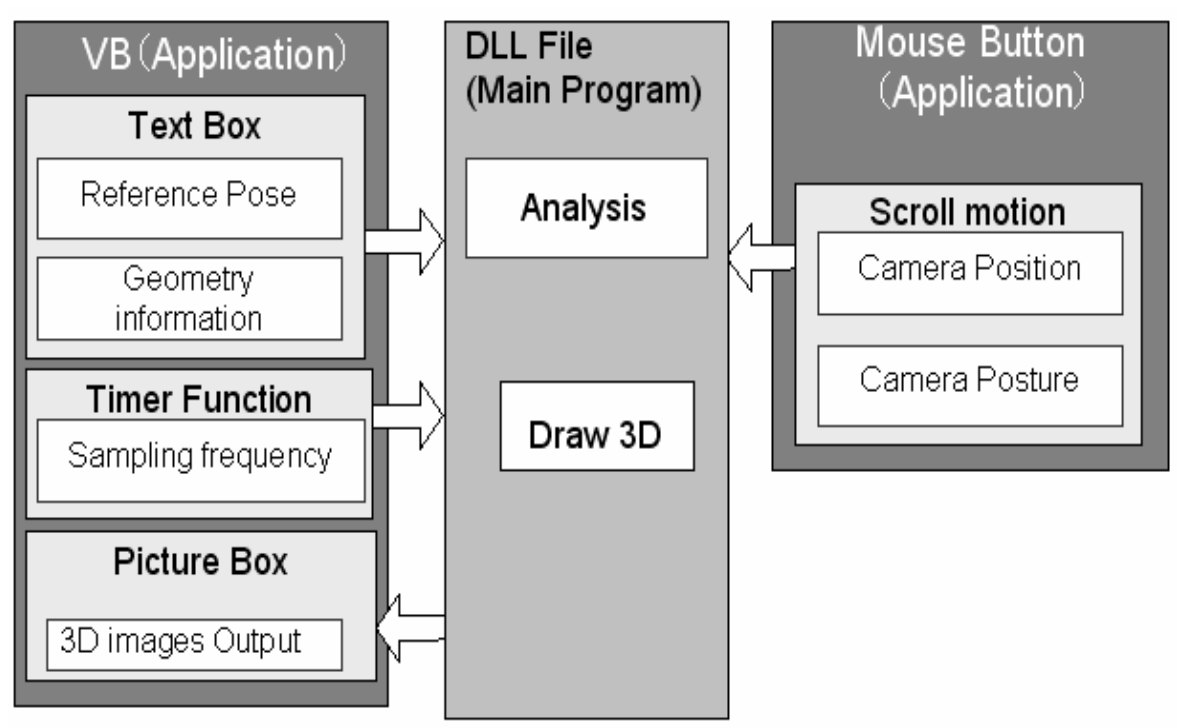

Fig.11 Main modules of Application 
Figure 12 shows the details of the DLL file system. VC++ first executes the workspace program based on the geometrical analysis, and then constructs the command that can draw $3 \mathrm{D}$ graphics. OpenGL provides the high-precision and high-speed rendering irrespective of OS on PC. $\mathrm{VC}++$ receives graphics data rendered by the OpenGL ${ }^{(5)}$, (6), which are then transferred to the application $(\mathrm{VB})$ in order to display on picture box.

The procedure followed to obtain a real-time workspace and the spatial movement of the parallel manipulator is the following. For a set of pose of the 6-DOF, i.e., $\left(x_{r}, y_{r}, z_{r}, \phi_{r}, \theta_{r}, \varphi_{r}\right)$, first the reference vector $\mathbf{R}$ (Eq. (4)) will generate the cylinder vector, the configurations of the platform and the cylinder. Then the workspace vector $\mathbf{R}$ successively calculates the arbitrary point on z-level cutting plane that satisfies the constraint conditions under the same set pose. The new configuration of the mechanism corresponding to the point $\mathbf{R}$ will be created for the graphical presentation on the picture box of VB. This computation process has been ported to the VB environment for a real-time coordination of workspace and spatial movement of the mechanism.

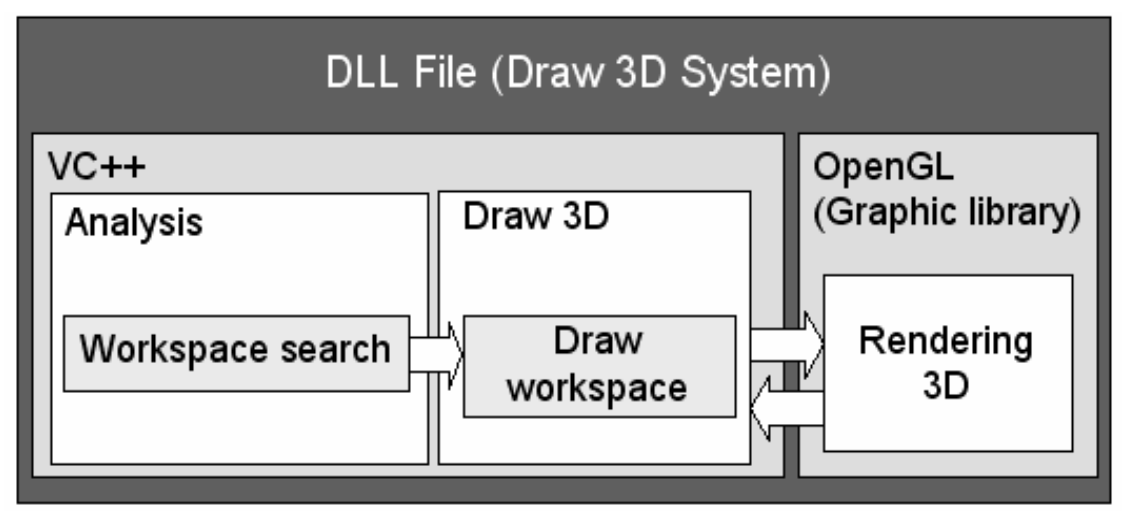

Fig.12 3D drawing system based on $\mathrm{VC}++$ and OpenGL

\subsection{Details of processing 3D-workspace display}

It is obvious from experiments that the actual workspace of the manipulator constitutes smooth surfaces. In analysis, the workspace vector $\mathbf{R}$ is estimated for the discrete values of rotation angle $\alpha$ and $\mathrm{z}$ level, i.e., $\Delta \alpha$ and $\Delta z$, which results in discrete vector $\mathbf{R}(\mathrm{i}, \mathrm{j})$. To construct the smooth surface of vector $\mathbf{R}$ whose coordinate is given by $x_{i j}, y_{i j}, z_{i j}$, two triangle planes are generated for the vectors $\mathbf{R}(i, j), \mathbf{R}(i, j+1), \mathbf{R}(i+1, j+1)$, and $\mathbf{R}(i+1, j)$, as shown in Fig.13.

The corresponding OpenGL commands are given in the following listing code.

\section{PROGRAM LISTING}

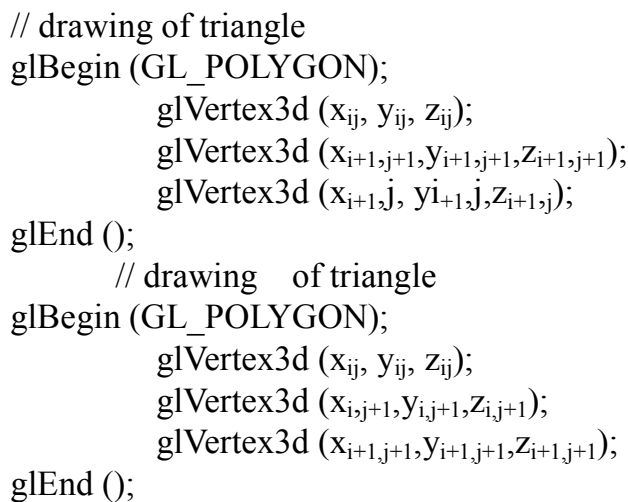




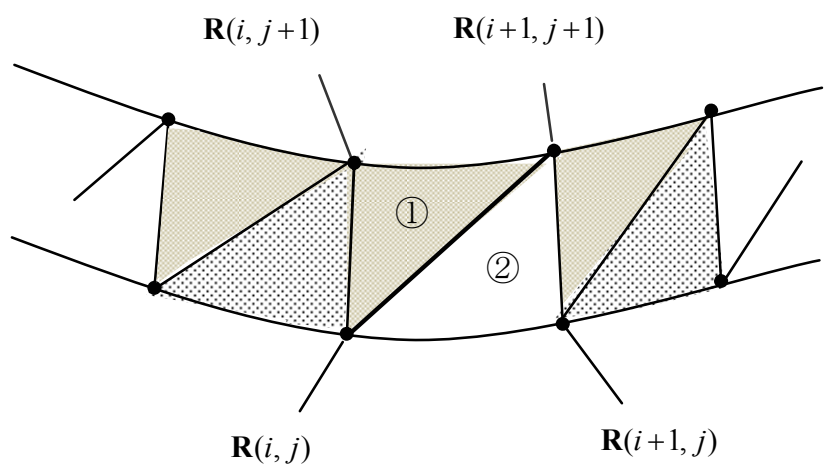

Fig.13 Surface triangular planes

Figure 14 shows the main screen developed with OpenGL technology for the 3D visualization of the workspace. The main function of this system is summarized as follows: 1) The main screen for $3 D$ display was designed with VB that facilitates inclusion of controls. The screen displays the 3D workspace and includes the control for data settings, where design parameters such as platform geometry and cylinder location as well as the6DOF poses of the manipulator can be easily set.

2) The display screen can be turned on and off at every $0.01 \mathrm{~s}$ using PC with clock $2.8 \mathrm{GHz}$, which provides enough speed to view the variation of workspace and continuous motion of the platform in a lifelike virtual reality environment. At the same time, 6-DOF poses can be

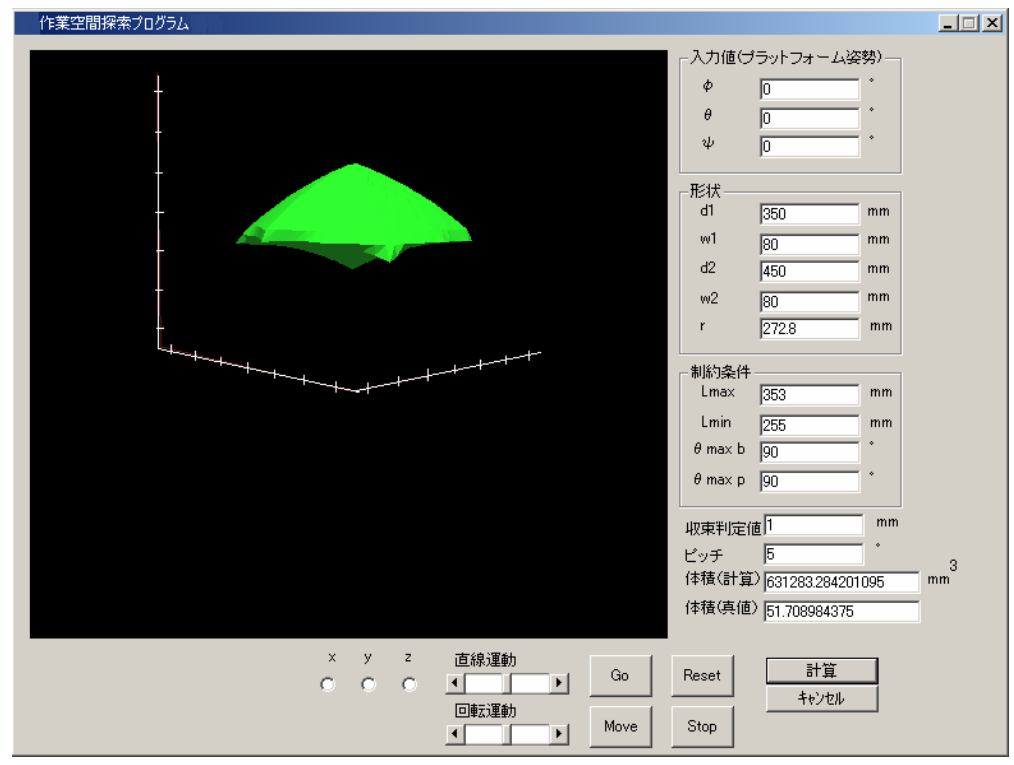

Fig.14 Main screen

read in screen when the workspace is displayed in real time.

3) The generated 3D workspace can be rotated around the 3 axes, and translated in perspective for better visualization of the details by scrolling mouse (Mouse Button Application).

Figure 15 shows an example view of workspace in 3-view mode when the platform is set at the neutral position. Figure 16 shows the effect of the platform pose on the workspace of the parallel manipulator under study. For a neutral position, the maximum cylinder lengths mainly define the upper half of the workspace, while the middle section of the workspace is free from the joint angle limits, and the bottom of the workspace is defined by minimum cylinder length. Figures 16 (a-1)-(a-2), (b-1)-(b-2), and (c-1)-(c-2) show the reduction of the workspace as the platform rolls, pitches, and yaws from 5 to 10 degrees, respectively. 
The general shape is similar to the neutral pose, but the bottom section of the workspace is reduced considerably and the vertices disappear at bottom with increase in platform posses. Since the movement of the working point of the manipulator occurs in 3D space, the boundary of the accessible workspace is a $2 \mathrm{D}$ surface.

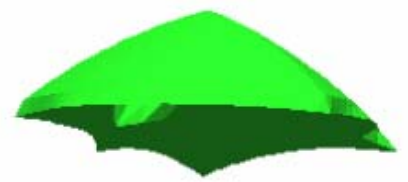

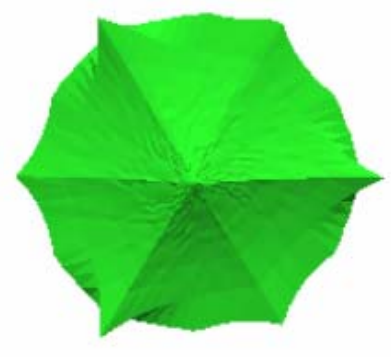

(b) Bottom

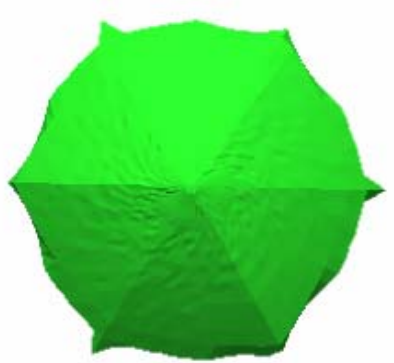

(c) Top

Fig. 15 View of workspace

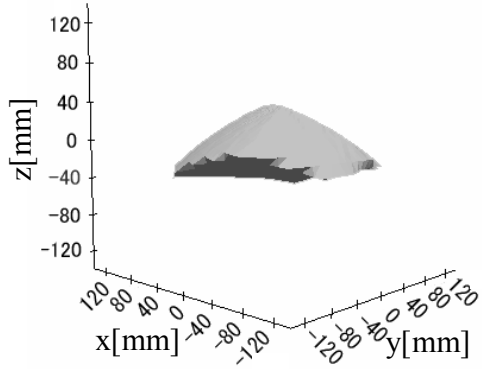

(a-1) $\phi=5^{\circ}$

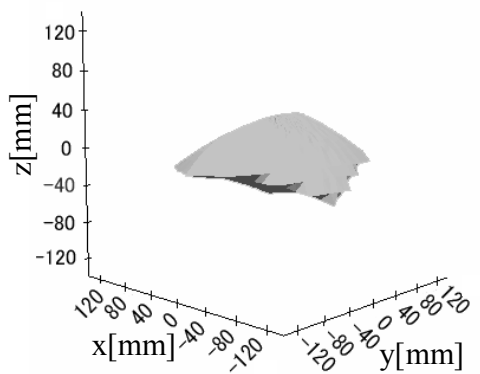

(b-1) $\theta=5$

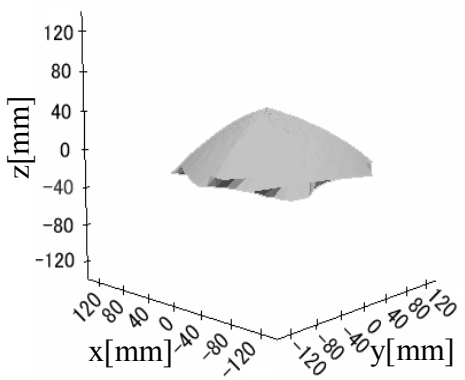

(c-1) $\phi=5^{\circ}$

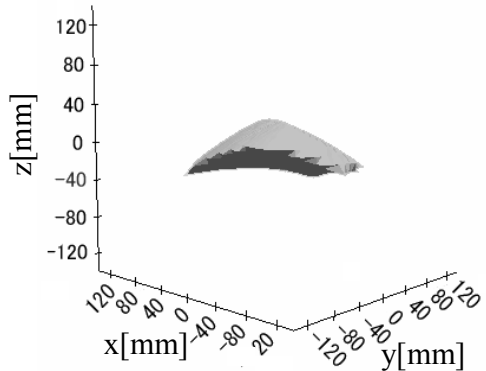

(a-2) $\phi=10^{\circ}$

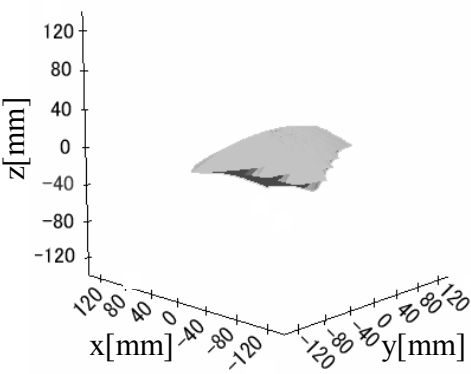

(b-2) $\theta=10^{\circ}$

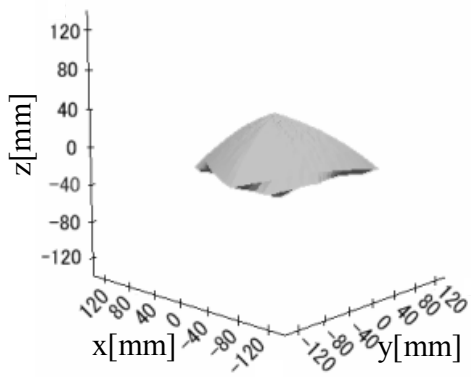

(c-2) $\phi=10^{\circ}$

Fig. 16 Effect of platform pose on the workspace configurations 


\section{Conclusions}

The inverse kinematics for the workspace analysis of parallel manipulator has been formulated, and a geometrical algorithm to evaluate the workspace has been developed using search vector and trajectories of cylinders on the z-level cutting plane that precisely define the workspace boundaries. The workspace constraint is formulated in terms of the cylinder length, with the mechanical interference, such as angular limits and collision between links, neglected. Workspace plots show that as the platform orientation increases, the overall workspace decreases. And comparison of the numerical search with the measurements showed good agreement. Also, this numerical search method to generate workspace, together with mechanism motion developed, is generic to be applied to other parallel-serial manipulator if the geometry of the machine, such as construction machines, is available. To provide design efficiency this computation process has been ported to VB environment for a real-time coordination of workspace plots

\section{Acknowledgement}

The research work reported in this paper is sponsored in part by the Japan Society for the Promotion of Science (JSPS) under Grant-in-Aid for Scientific Research (C), No. 20500500 .

\section{Nomenclature}

$\mathrm{O}$ : centroid $\mathrm{O}$ of the base

O' : centroid O' of the platform

$\mathbf{R}$ : position of the platform

$\mathbf{A}_{i}$ : position of the attachment point of the cylinder on the platform

$\mathbf{B}_{i}$ : position of the attachment point on the base

$\mathbf{A}_{\text {im }}$ : position vectors for the platform joint

$\ell_{i}, L_{i}$ : cylinder vector and its length

$\mathrm{x}, \mathrm{y}, \mathrm{z}:$ motions for surge, sway, and heave

$\phi, \theta, \phi:$ angles for roll, pitch, and yaw

\section{References}

(1) S. Ibaraki, T. Okuda, Y. kakino, M. Nakagawa, T. Matsushita, Disturbance Estimation on a Hexapod-Type Parallel Kinematic Machine Tool by Using A Disturbance Observer, Transactions of the Japan Society of Mechanical Engineers, Series C, Vol.70, No.694 (2004), pp.1771-1776.

(2) J. P. Conti, C. M. Clinton, G. Zhang, and A. J. Wavering, Workspace Variation of a Hexapod Machine Tool, National Institute of Standards and Technology (NIST) 6135 (1998), pp.1-15.

(3) C. Gosselin, Determination of the Workspace of 6-DOF Parallel Manipulators, Transactions of the ASME, Journal of Mechanical Design, Vol.112 (1990), pp.331-336.

(4) D. Liu, R. Che, Z. Li, and X. Luo, Research on the Theory and the Virtual Prototype of 3-DOF Parallel-Link Coordinate-Measuring Machine, IEEE Transactions on Instrumentation and Measurement, Vol.52, No.1 (2003), pp.119-125.

(5) Z. Zhang, S. Ma, B. Li, L. Zhang, and B. Cao, Development of an OpenGL Based Multi-robot Simulating Platform, $8^{\text {th }}$ International Conference on Control, Automation, Robotics and Vision Kunming, China (2004), pp.400-1405.

(6) F. Kober and F. A. B. Lemons, On Use of OpenGL Technology to Support a Power System CAD, IEEE PES Power Systems Conference and Exposition (2004), pp.995-999. 